\title{
Chondroprotection by histone deacetylase inhibition
}

Histone deacetylases (HDACs) regulate transcription, including that of genes encoding matrix metalloproteinases (MMPs). The possibility of using HDAC inhibitors (HDACi) to treat osteoarthritis (OA) via repression of cartilage-degrading MMP expression has now been advanced by work published in Arthritis \& Rheumatism by Ian Clark and colleagues.

Previously, broad-spectrum HDACi were found to block cartilage destruction in vitro and were effective in mouse models of inflammatory arthritis. Inflammation has a minimal role in the destabilization of the medial meniscus (DMM) mouse model of OA, which, says Clark, "is the most common model of OA used currently. HDACi haven't been tested in this model before."

Systemic delivery of trichostatin A, a broad-spectrum HDACi, over 8 weeks inhibited OA in DMM mice, presumably through suppression of MMP expression. Nevertheless, the toxicity profiles of

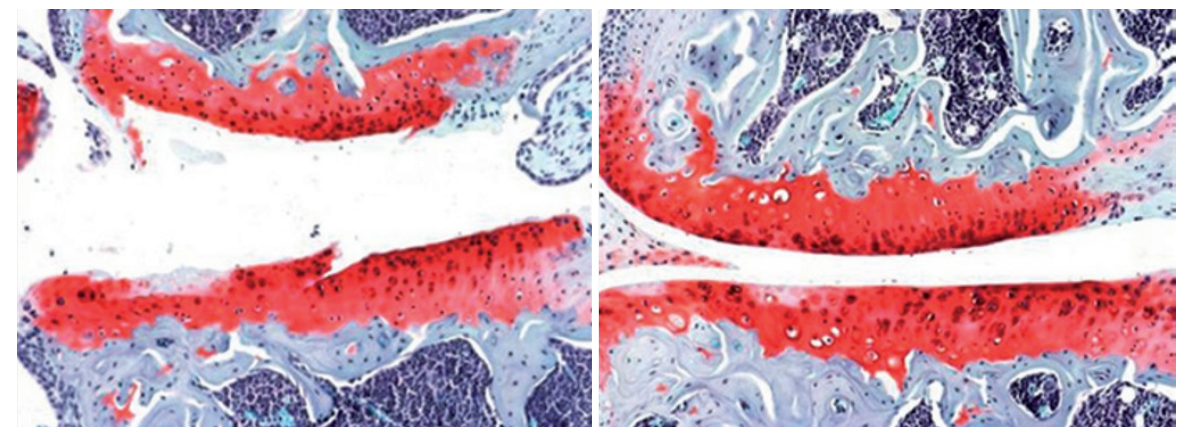

Representative medial sections from most affected knee joint in mice with osteoarthritis induced by surgical meniscal destabilization, after 8 weeks of treatment with vehicle (left) or trichostatin A (right). Image courtesy of David Young and Wang Hui.

broad-spectrum HDACi make them unsuitable for use in chronic, non-life threatening conditions. Thus, "we tested if inhibitors selective for sub-groups of HDACs could work," continues Clark. Using primary human articular chondrocytes and bovine cartilage explant assays, the investigators found that class I HDAC-selective compounds mimicked the action of broadspectrum HDACi in vitro; the approach might thus be expected to work in vivo.
Developing selective HDACi and testing them in vivo are ongoing challenges. Favourable results "may allow the development of less toxic drugs for use in OA," suggests Clark.

\section{Emma Leah}

Original article Culley, K. L. et al. Class I HDAC inhibition modulates metalloproteinase expression and blocks cytokine-induced cartilage degradation. Arthritis Rheum. doi:10.1002/art.37965 